\title{
Die dunkle Seite der Macht
}

\section{Fallbeispiele negativer spiritueller Erfahrungen}

\author{
Stefan Huber $(\mathbb{D}) \cdot$ Mathias Tanner $(\mathbb{D}) \cdot$ Herbert Scheiblich
}

Eingegangen: 20. November 2020 / Angenommen: 4. Dezember 2020 / Online publiziert: 7. Januar 2021 (C) Der/die Autor(en) 2021

Zusammenfassung Der Begriff Spiritualität ist in gesellschaftlichen und wissenschaftlichen Diskursen fast ausschließlich positiv konnotiert und wird primär mit Themen wie Gesundheit, Wohlbefinden, Lebenszufriedenheit oder Intelligenz in Verbindung gebracht. Im Kontrast dazu werden im vorliegenden Beitrag vier Fallbeispiele diskutiert, in denen Erzählungen zu negativen Erfahrungen oder Einstellungen zu Spirituellem erzählt werden. Die Erzählungen stammen aus Leitfadeninterviews, die im Rahmen einer repräsentativ angelegten „mixed methods“ Studie zu Säkularen und Säkularität in der Schweiz durchgeführt wurden. Rund vier Jahre nach den Erstinterviews konnten mit drei der Befragten Zweitinterviews durchgeführt werden. In beiden Interviews wurden die Auskunftspersonen unter anderem gefragt, ob es in ihrem Leben etwas Spirituelles gebe. Darauf erzählten sie von Schicksalsmächten, „Geistern“, „schwarzer Magie“ oder „schlechter Energie“, die bei ihnen und nahestehenden Personen Wünsche erfüllt (was später negativ bewertet wurde), negative Gefühle wie Angst, Kälte oder Leere ausgelöst oder dann auch Krankheiten, Streit, schlimme Vorfälle oder unerklärliche Unfälle verursacht hätten. In der Diskussion werden mögliche Konsequenzen dieser Befunde für die Spiritualitätsforschung und den Begriff der Spiritualität reflektiert. Dabei werden Verbindungen zu Forschungen über außergewöhnliche Erfahrungen - die weit verbreitet sind hergestellt und Anregungen für weitere Forschungen gegeben. In Bezug auf die Begriffsdiskussion plädiert der Beitrag dafür, Spiritualität konsequent auf Erfahrungen

\footnotetext{
S. Huber $(\bowtie) \cdot$ M. Tanner $\cdot$ H. Scheiblich Institut für Empirische Religionsforschung, Theologische Fakultät, Universität Bern, Länggassstrasse 51, 3012 Bern, Schweiz

E-Mail: stefan.huber@theol.unibe.ch

M. Tanner

E-Mail: mathias.tanner@theol.unibe.ch

H. Scheiblich

E-Mail: herbert.scheiblich@theol.unibe.ch
} 
„großer Transzendenzen“ im Sinne Luckmanns zu beziehen. Dadurch wird der Begriff für negative spirituelle Erfahrungen geöffnet. Daneben ergeben sich dadurch neue Impulse für die Bestimmung des Verhältnisses von Spiritualität und Religion.

Schlüsselwörter Begriff der Spiritualität · Negative spirituelle Erfahrungen · Außergewöhnliche Erfahrungen · Säkulare · Religiosität · Schwarze Magie · Geister

\section{Negative experiences with spirituality}

Case reports

Abstract In social and scientific discourses the term spirituality almost exclusively has a positive connotation and is primarily associated with topics such as health, well-being, life satisfaction or intelligence. In contrast, this article discusses four case studies in which stories about negative experiences or attitudes towards spirituality are told. These stories are taken from guideline interviews that were conducted as part of a representative mixed methods study on secular people and secularism in Switzerland. About four years after the first interviews, second interviews could be conducted with three of the respondents. In both interviews the respondents were asked, among other things, whether there was something spiritual in their lives. Then they told about forces of fate, "ghosts", "black magic" or "bad energy" that fulfilled wishes (which was later evaluated negatively), triggered negative feelings such as fear, cold or emptiness, or then also caused illness, quarrels, bad incidents or inexplicable accidents. The discussion reflects on the possible consequences of these findings for spirituality research and the concept of spirituality. Connections are made to research on extraordinary experiences_-which are widespread—and suggestions for further research are given. With regard to the discussion of terms, this article pleads for spirituality to be consistently related to experiences of "great transcendences" in Luckmann's sense. This opens the term to negative spiritual experiences. In addition, new impulses for the definition of the relationship between spirituality and religion arise from this.

Keywords Concept of spirituality $\cdot$ Negative spiritual experiences $\cdot$ Extraordinary experiences $\cdot$ Secular people $\cdot$ Religiosity $\cdot$ Black magic $\cdot$ Ghosts

Résumé Dans les discours sociaux et scientifiques, le terme de spiritualité est presque exclusivement connoté de façon positive et est principalement associé à des sujets tels que la santé, le bien-être, la satisfaction de vivre ou l'intelligence. En revanche, cet article traite de quatre études de cas dans lesquelles sont relatées des expériences ou des attitudes négatives à l'égard de la spiritualité. Les récits sont tirés d'entretiens guidés qui ont été menés dans le cadre d'une étude représentative «de méthodes mixtes» sur les laïques et la laïcité en Suisse. Environ quatre ans après les premiers entretiens, des seconds entretiens ont été menés avec trois des personnes interrogées. Dans les deux entretiens, on a demandé aux personnes interrogées, entre autres, s'il y avait quelque chose de spirituel dans leur vie. En réponse, ils ont parlé des forces du destin, des «esprits», de la «magie noire» ou de la «mauvaise énergie» qui ont réalisé leurs souhaits et ceux de leurs proches (ce qui a été évalué négati- 
vement par la suite), ont déclenché des sentiments négatifs tels que la peur, le froid ou le vide, ou ont aussi provoqué des maladies, des querelles, de mauvais incidents ou des accidents inexplicables. La discussion porte sur les conséquences possibles de ces résultats pour la recherche sur la spiritualité et le concept de spiritualité. Des liens seront établis avec les recherches sur les expériences extraordinaires - qui sont largement diffusées - et des suggestions pour des recherches supplémentaires seront données. En ce qui concerne la discussion des termes, l'article plaide pour que la spiritualité soit systématiquement liée aux expériences de «grandes transcendances» au sens de Luckmann. Cela ouvre le terme à des expériences spirituelles négatives. En outre, de nouvelles impulsions pour la définition de la relation entre la spiritualité et la religion en découlent.

Mots-clés Concept de spiritualité · Expériences spirituelles négatives · Expériences extraordinaires · Laïques · Religiosité · Magie noire · Esprits

\section{Einleitung}

Der Begriff „Spiritualität“ ist in sozialen Diskursen fast durchgängig mit positiven Konnotationen verbunden und wird dabei oft in Abgrenzung zu den Begriffen „Religion“ und „Religiosität“" verwendet, die dadurch als das Negative des Spirituellen erscheinen. Dies spiegelt sich in empirischen Studien, die den alltäglichen Gebrauch dieses Wortes untersuchen (Altmeyer et al. 2015; Demmrich und Huber 2019, im Druck; Eisenmann et al. 2016; Tong und Yang 2018). Analoge Tendenzen lassen sich in wissenschaftlichen Diskursen beobachten, die Spiritualität im Kontext von Gesundheit, Wohlbefinden, Lebenszufriedenheit und Intelligenz thematisieren (vgl. Emmons 2000; Koenig 2008, 2011; Zinnbauer und Pargament 2005).

Doch werden spirituelle Erfahrungen tatsächlich immer nur als positiv, d.h. als angenehm und förderlich erlebt? Gibt es auch Fälle, in denen Spiritualität als unangenehm, bedrohlich oder destruktiv erlebt wird? Was folgt daraus für die zukünftige Forschung und die begriffliche Bestimmung von Spiritualität? Diesen Fragen soll im vorliegenden Beitrag auf der Basis von vier Fallbeispielen nachgegangen werden.

Um im Folgenden den Bereich des Spirituellen einzugrenzen, orientieren wir uns an der Arbeitsdefinition, die Bucher (2014) vorgeschlagen hat.

$\mathrm{Er}$

,plädiert für ein breites Verständnis von Spiritualität, deren Kern Verbundenheit ist, zum einen horizontal mit der sozialen Mitwelt, der Natur und dem Kosmos, zum anderen vertikal mit einem den Menschen übersteigenden, alles umgreifenden Letztgültigen, Geistigen, Heiligen, für viele nach wie vor Gott. Diese Öffnung aber setzt voraus, dass der Mensch auch zu Selbsttranszendenz fähig ist und vom eigenen Ego absehen kann." (Bucher 2014, S. 69)

Unserer Ansicht nach ist dabei die menschliche Fähigkeit der Selbsttranszendenz im Sinne des Begriffs der „exzentrischen Positionalität“ (Plessner 1928) und der Vergesellschaftung des Umgangs mit Transzendenzerfahrungen (Luckmann 1991) fundamental. In dieser Linie erscheint Spiritualität als ein anthropologisch und so- 
ziologisch tief verankertes Phänomen. Auch aus diesem Grund ist es sinnvoll, die Frage nach negativen spirituellen Erfahrungen zu stellen.

\section{Stichprobe und Methode}

Die vier Fälle, auf die wir im Folgenden eingehen, stammen aus einer Teilstichprobe eines von Stefan Huber und Jörg Stolz geleiteten und vom Schweizerischen Nationalfonds finanzierten Projekts zu Säkularen in der Schweiz. ${ }^{1}$ Ausgangspunkt war die repräsentative Umfrage des Religionsmonitors in der Schweiz $(n=1003)$, die im November und Dezember 2012 durchgeführt wurde. Während der computergestützten Telefonumfrage wurden Teilnehmende, die sich im Interview als ,gar nicht religiös“ oder zumindest als ,eher atheistisch“ charakterisiert haben (341 Teilnehmende, $34 \%$ der Stichprobe), gefragt, ob sie zu einem vertiefenden Interview bereit wären. Dies bejahten 113 Personen (33\% der Kategorie). Aus dieser Gruppe wurden 83 Personen nach einer Schichtung in Bezug auf die Kategorien Alter, Geschlecht, Sprache und konfessionelle Zugehörigkeit per Zufall für Interviews ausgewählt. Von diesen Interviews wurden 48 auf Deutsch geführt. Diese 48 Interviews wurden bereits von Demmrich und Huber (2019) in Bezug auf das Spiritualitätsmodell von Bucher (2014) sowie das Religiositätsmodell von Huber (2003) analysiert. Dabei zeigte sich, dass sich beide Modelle gut dafür eignen, Erzählungen von Säkularen zur Spiritualität und zu Spirituellem in strukturierter Weise abzubilden. Weiter konnten Demmrich und Huber (im Druck) auf der Basis dieser Interviews das von Eisenmann et al. (2016) induktiv gewonnene Kategoriensystem subjektiver Definitionen der Spiritualität replizieren. Dabei zeigte sich, dass es sich auch zur Beschreibung der Spiritualität von Säkularen eignet.

Die 48 vertiefenden Interviews standen im Kontext eines Forschungsprojekts zu Selbstverständnis, Werten, Zielen und Engagements von Säkularen und wurden in den Jahren 2013 und 2014 geführt. In den Leitfadeninterviews wurden Fragen zum persönlichen und familiären Leben, der Zugehörigkeit zu Gruppen, Vereinen oder Clubs sowie zur säkularen Identität gestellt. Gegen Ende des Interviews wurden die Themen Lebenssinn, alternative Heilmethoden und Spiritualität (,Gibt es etwas Spirituelles in Ihrem Leben?“) angesprochen. Danach folgten Fragen zu Religiosität (,Gibt es etwas Religiöses in Ihrem Leben?“) und Kirchen (,Welche Erfahrungen haben Sie mit Kirchen oder anderen religiösen Gemeinschaften gemacht?").

Rund vier Jahre später konnten mit 35 Personen Zweitinterviews geführt werden. Diese Leitfadeninterviews fanden nicht mehr im theoretischen Rahmen des Forschungsprojekts zur Säkularität statt. Stattdessen richtete sich das Erkenntnisinteresse nun auf Spiritualität und Religiosität. Nach der Eingangs- und Aufwärmfrage zu wichtigen Lebensereignissen seit dem Erstinterview wurden diesmal zunächst die Themenbereiche Spiritualität (,Ist in den letzten Jahren etwas Spirituelles in Ihrem Leben vorgekommen?"), Religiosität sowie Kirchen und Religionsgemeinschaften angesprochen. Erst danach richtete sich das Interview auf die anderen Themenbereiche des Erstinterviews.

\footnotetext{
${ }^{1}$ Siehe: http://p3.snf.ch/Project-156241.
} 
In vier Interviews, die wir im Folgenden vertieft analysieren, sprechen die Auskunftspersonen negative Aspekte der Spiritualität an. Eine Person erzählte dies im Erstinterview. Drei Personen kamen darauf erst im Zweitinterview zu sprechen.

\section{Empirische Befunde}

Die Tab. 1 präsentiert Basisdaten zu den vier Leitfadeninterviews mit negativen spirituellen Erfahrungen sowie zu ausgewählten Variablen der quantitativen Befragung. Zunächst wird jeweils der Durchschnittswert der 48 Interviews aufgeführt, dann folgen die Werte zu den vier Fällen. Bei den Fallnamen handelt es sich um Pseudonyme.

In den folgenden Abschnitten sprechen die vier Interviewten über ihre negativen spirituellen Erfahrungen. Dabei dokumentieren wir alle relevanten Stellen aus dem Interview. Die Originalaussagen werden durch Kursivschrift hervorgehoben. Weiter werden in Klammern Parasprachliches (z. B. lacht) sowie Betonungen durch Großbuchstaben und Pausen durch Punkte oder Sekundenangaben dokumentiert.

\subsection{Ariane - „Wünsche an das Universum“6}

Im Erstinterview hatte Ariane erzählt, dass sie „Wünsche an das Universum“ richte, „solche Sachen, wenn man sich (...) etwas wünscht, dann wird das irgendwann in Erfüllung gehen“. Das habe für sie ,auch eine gewisse Spiritualität, weil es in das Esoterische geht". Demgegenüber bewertet sie dieses Wünschen im Zweitinterview negativ: „Da sind alle meine Wünsche in Erfüllung gegangen. Und das habe ich

Tab. 1 Basisdaten zu den vier Leitfadeninterviews und der Stichprobe aus der sie stammen

\begin{tabular}{|c|c|c|c|c|c|}
\hline \multirow[b]{2}{*}{$\begin{array}{l}\text { Qualitative } \\
\text { Interviews }\end{array}$} & \multirow{2}{*}{$\begin{array}{l}\text { 48/35 Fälle } \\
\varnothing \\
-\end{array}$} & \multicolumn{4}{|c|}{ Vier Einzelfälle } \\
\hline & & Ariane & Antonio & Gabriel & Martin \\
\hline $\begin{array}{l}\text { 1. Interview, } \\
\text { Zeitpunkt }\end{array}$ & $2013 / 14$ & Juni 2013 & Jan. 2014 & Jan. 2014 & Nov. 2013 \\
\hline Länge & $77 \mathrm{~min}$ & $90 \mathrm{~min}$. & $49 \min$ & $56 \min$ & $40 \mathrm{~min}$ \\
\hline $\begin{array}{l}\text { 2. Interview, } \\
\text { Zeitpunkt }\end{array}$ & $2017 / 18$ & Nov. 2017 & Nov. 2018 & $\begin{array}{l}\text { Kein Inter- } \\
\text { view, da }\end{array}$ & März 2018 \\
\hline Länge & $72 \mathrm{~min}$ & $155 \mathrm{~min}$ & $60 \mathrm{~min}$ & verstorben & $55 \mathrm{~min}$ \\
\hline Quantitative Studie & 48 Fälle $\varnothing$ & Ariane & Antonio & Gabriel & Martin \\
\hline Alter (2012) & 51 & 50 & 46 & 21 & 47 \\
\hline $\begin{array}{l}\text { Geschlecht } \\
\text { (männlich) }\end{array}$ & $73 \%$ & Weiblich & Männlich & Männlich & Männlich \\
\hline Bildung (Universität) & $65 \%$ & Gymnasium & Berufsschule & Berufsschule & Universität \\
\hline $\begin{array}{l}\text { Ökonomische Lage } \\
(1-4)\end{array}$ & 3,2 & Sehr gut (4) & $\begin{array}{l}\text { Eher } \\
\text { gut (3) }\end{array}$ & Eher gut (3) & Sehr gut (4) \\
\hline Gesundheit (1-4) & 3,2 & Sehr gut (4) & $\begin{array}{l}\text { Eher } \\
\text { gut (3) }\end{array}$ & Eher gut (3) & $\begin{array}{l}\text { Eher } \\
\text { gut (3) }\end{array}$ \\
\hline
\end{tabular}


teilweise teuer bezahlt. (..) Wissen Sie, die Wünsche, die man manchmal hat, die sind nicht immer zu seinem Besten. (..) Finde ich jetzt.“ Dazu erklärt sie: „Es ist nicht immer gut, wenn man immer selber bestimmen kann, was man sich wünscht (..) und es geht dann in Erfüllung, weil man muss es sich dann nicht erarbeiten; es geht dann in Erfüllung, und dann hat man es dann. Und das kann dann unter Umständen (.) gar nicht so gut sein für einen.“ Weiter erklärt sie: „Ja es war auf den ersten Blick schon gut, aber ich habe nachher in der Konsequenz am Schluss in der Endabrechnung musste ich sagen: Eigentlich will ich das nicht mehr. Ich will nicht MIR etwas wünschen, sondern es soll PASSIEREN. (..) Sonst, wenn ich mir etwas wünsche, dann WARTE ich auch darauf. Und wenn es dann kommt, dann kommt ja noch meine Interpretation dazu und meine Begeisterung, denn ich habe ja etwas gehofft. Und wenn man etwas/das ist wie ein Schönsaufen (lacht). Von dem, was nachher kommt, oder. Denn das habe ich mir ja gewünscht! Jetzt nehme ich mir das! Oder. Und vielleicht hat man ursprünglich schon noch zwei, drei andere Ecken und Kanten gesehen, von denen man gesagt hat: ,Ja, aber so dann nicht. 'Aber wenn es dann da ist, dann nimmt man es, oder. Man hat es dann gewünscht. Man ist dann in diesem Modus drin. Und da musste ich sagen: ,Nein, das will ich gar nicht mehr'. (..) Ich habe dieses Buch auch weggeworfen (lacht).“

\subsection{Antonio - „Geister“ und „Leuten schaden“"}

Im Erstinterview assoziiert Antonio Spirituelles in seinem Leben mit Naturerfahrungen: „Ich bin sehr gern in der Natur. Das ist so ein bisschen meine Spiritualität. Ich bin sehr gern im Wald und so, ganz allein. Ich gehe auch immer (zeigt mit der Hand:) Da hinten habe ich mein Aussichtsplätzchen und so. Oder auf unserer Alp oben. Dort fühle ich mich eigentlich so ein bisschen spirituell geborgen.“ Und: „Möglichst wenig Menschen, das ist meine Spiritualität (lacht).“ Im Zweitinterview erzählt Antonio wieder direkt nach der Frage zu Spirituellem in seinem Leben - eine Angsterfahrung, die er bei seiner Alphütte, die er im Erstinterview positiv erwähnt hatte, erlebt hat: „Also gestern (...) ist (..) also etwas ganz Komisches gewesen. Gestern wollte ich in die Alp hinein. Ich konnte nicht hinein. Es war ganz komisch." Auf Nachfragen fügt er folgende Beschreibungen hinzu: „Ich konnte nicht. Ich musste gleich wieder raus. Etwas war nicht gut. Da bin ich gleich wieder gegangen. (...) Das habe ich jetzt schon lange nicht mehr erlebt, so etwas. (...) Dubios gewesen. Ja-ha-hatte Angst hinein zu gehen, einfach (...). Das hatte ich eher in der Jugend. Und gedacht etwas stimme da nicht, bin gleich wieder raus. (lacht) Dubios gewesen." Als Erklärung verweist er auf ähnliche Erfahrungen in seiner Jugendzeit: „Ja, ich hatte das in der Jugend $a b$ und zu. Ich weiß auch nicht, (..) ob da irgendetwas da ist, irgendwelche (lacht) Geister oder ich weiß auch nicht." Das Thema abschließend betont Antonio: „Aber ich tue mich jetzt nicht gro $\beta$ damit beschäftigen. Ich bin einfach wieder gegangen, und fertig (lacht)." Danach fragt der Interviewer nach weiteren Beispielen, die er als spirituell bezeichnen würde. Antonio: „Nein, ja, ich denke ich kann den Leuten eher schaden als nützen, wenn ich will. Wenn ich wirklich wütend bin (lacht). Danach merke ich, dass es den andern nicht gut geht, oder (...). Wobei, das ist auch schon lange nicht mehr vorgekommen (lacht). Also wenn mich irgendein Arbeitskollege oder so so richtig aufregt, dann ist der garantiert nächste Woche krank. Das 
habe ich inzwischen gemerkt. Ich habe ihnen nichts Schlechtes gewünscht. Aber es ist einfach selber schon (...). Nur schon (...). Nur schon, dass ich mich aufregte hat offenbar gereicht."

\subsection{Gabriel - ,schwarze Magie“6}

Gabriel erzählt direkt nach der Frage zu Spirituellem in seinem Leben negative Erfahrungen: „Ja, das hatte ich schon erlebt. Definitiv. Aber das war nicht so lustig, sagen wir es so (lacht). Nein, das war wirklich nicht mehr so lustig. Die eine ExFreundin, die hat immer wieder von so Dingen erzählt. Und dann sind wirklich Dinge passiert, von denen ich mir eigentlich auch nicht mehr erklären konnte, ob es mit rechten Dingen zugeht oder menschlich ist." Er nennt einige Ereignisse, die er mit der Ex-Freundin erlebt hat: „Bei der einen Ex-Freundin war es zum Beispiel so: Sie hatte eigentlich dunkelbraune Augen. Und sie hatte so Phasen. Wenn du ihr dann in die Augen geschaut hattest: Erstens war sie nicht mehr ansprechbar.“ Weiter: „Und wenn du ihr dann in die Augen schautest, dann waren die Augen wirklich hellbraun. Und ich bin nicht farbenblind oder sonst irgendetwas. Wenn du das irgendjemandem erzählst, dann: hä? Aber das waren dann jeweils so Dinge, dann ging es fünf, zehn Minuten, dann sackte sie zusammen. Wie ein Sack Kartoffeln. Und dann kam sie wieder zu sich. Dann konntest du wieder normal mit ihr sprechen. Und ihre Augen waren wieder schön dunkelbraun.“ Und: „Oder du lagst im Bett mit ihr. Und es war eigentlich 25 Grad in dem Zimmer. Und du warst nicht krank. Aber plötzlich begannst du zu frieren, und dir war kalt, du fühltest dich leer. Und bösere Sachen, sagen wir es so. Mehr schwarze Magie. So etwas. Oder dass Fenster auf und zu gingen, wo du eigentlich sagtest: Du, es gab keinen Luftzug, nichts, keinen Durchzug, wirklich nicht. Und die Türen gingen auf und zu. Fenster gingen auf und zu. Es knalle im Haus drin. Solche Dinge sind mir passiert, echt." Einer vertieften Auseinandersetzung mit diesen Phänomenen wich Gabriel letztendlich aus: „Es interessierte mich dann auch, was denn eigentlich genau dahintersteht. Was denn das eigentlich ist. Aber im Großen und Ganzen habe ich die Finger davon gelassen. Weil ich wusste - ich habe es bei ihr gesehen -, dass es nicht gut war (...) Irgendwo macht dich das kaputt. Deswegen ließ ich es sein."

\subsection{Martin - ,okkulte Praktiken“" und ,schlechte Energie“6}

Im Zweitinterview berichtet Martin auf die Einstiegsfrage zu wichtigen Ereignissen, die sich in den letzten Jahren zugetragen haben, dass er unter einer akuten Psychose leide und seit Langem eine schizoaffektive Störung habe. Kurz nach der akuten Psychose sei er für etwa ein Jahr einer Freikirche beigetreten. Der Kontakt mit der Freikirche sei zustande gekommen wegen okkulter Praktiken, die seiner Ansicht nach früher auf dem Gelände, auf dem auch sein Haus steht, vorgekommen seien. Martin: „(...) Auf dem Gelände hier hat man okkulte Praktiken praktiziert, zum Beispiel pendeln und Karten legen. Und ich war der Meinung, dass hat einen schlechten Einfluss auf das Familienleben, was auch so war. Wir stritten oft. "Auf die spiegelnde Rückfrage präzisiert Martin: „Auf dem Gelände, als die Häuser noch nicht standen. Das ist eine Überbauung, die jemandem gehörte, der hier unten lebt. 
Einem älteren Herrn. Und der machte das, er vollzog okkulte Praktiken. Und ich habe da mit einem ... also angesprochen hat mich ein Mitbewohner dieser Siedlung. Er sagte, da stimme etwas nicht auf diesem Gelände. Das ist ganz schlechte Energie da. Wir streiten oft. Auch die Polizei war schon hier. Wirklich schlimme Sachen, schlimme Vorfälle. Und Unfälle gab es, ganz unerklärliche. Und dann sagten wir, da stimmt etwas nicht, das muss man auflösen. Dann diskutierten wir darüber, wie wir das machen könnten: katholischer Priester oder reformierter Priester. Und ich kam dann darauf, dass die Freikirche in A. einen Dienst anbietet speziell für Befreiung von Geländen, wo okkulte Praktiken vollzogen worden sind. Und so kam ich auf die Idee, die Freikirche in A. zu engagieren. Die kamen dann auch, und seither ist eigentlich Ruhe." Auf die Frage, was dabei geschehen sei, berichtet Martin sehr knapp, fünf, sechs Leute hätten die Häuser und das Gelände frei gebetet.

\section{Interpretative Vertiefung der vier Fälle}

Bei der interpretativen Vertiefung und Verdichtung der vier Erzählungen orientieren wir uns an dem Spiritualitätsmodell von Bucher (2014) sowie an dem multidimensionalen Modell der Religiosität von Huber (2003). Beide Modelle haben sich bereits bei der Analyse der Spiritualität der 48 Fälle der Ausgangsstichprobe als produktiv erwiesen (Demmrich und Huber 2019).

Ariane fühlt sich in der Praxis des Wünschens mit dem Universum verbunden, was sie zunächst fasziniert und begeistert. In späteren Reflexionen bewertet sie diese Art der Verbundenheit jedoch negativ und distanziert sich klar davon. Arianes Zuwendung zur Praxis des Wünschens ereignet sich in der Dimension der privaten Praxis, die von der intellektuellen Dimension im Sinn einer spirituellen Suche geführt wird. Die intellektuelle Dimension ist dann auch bei der Abwendung von dieser Praxis leitend (,Endabrechnung“), was zu einer Positionierung in der ideologischen Dimension führt (,es ist nicht immer gut"). Antonio erlebt eine Situation, in der er mit „etwas“ verbunden ist, was ,nicht gut“" ist und was auch „Geister“ sein könnten. In diesem Erzählkontext erinnert er sich an frühere Ereignisse, in denen er so stark mit von ihm als negativ wahrgenommenen Personen verbunden war, dass ihnen bereits seine innere Erregung ,schaden“ konnte. Die Erzählung dieser Ereignisse konzentriert sich eindeutig auf die Dimension der Erfahrung. Reflexionen werden nur ansatzweise thematisiert. Ebenso fehlt eine eindeutige ideologische Positionierung (,dubios“), was vermutlich mit seiner Verdrängung dieser Ereignisse zusammenhängt. Gabriel beobachtet an seiner Freundin, wie sie mit einer Macht verbunden ist, welche ihr negativ zusetzt und welche auch er selbst - vermittelt über sie - in Form von Kälte- und Leeregefühlen körperlich wahrnimmt. Wie bei Antonio steht die Dimension der Erfahrung im Zentrum. Gabriel nimmt Ereignisse wahr, die er davor nicht für möglich gehalten hatte. Daher führen diese Erfahrungen zu einer ideologischen Neupositionierung, er hält eine spirituelle Welt nun ,definitiv“ für real und definiert den Zugang zu ihr als ,schwarze Magie“. Die Entdeckung dieser neuen Welt stimuliert auch sein intellektuelles Interesse, doch er geht diesem Interesse nicht nach, sondern entscheidet sich wie Antonio für eine Strategie des Meidens dieses Bereichs. Martin interpretiert negative Vorfälle in seiner Siedlung im Licht 
der Konzepte „schlechte Energien“" und „okkulte Praktiken“. Er versucht, die Verbundenheit mit diesen Kräften durch ein „frei beten“, das von religiösen Spezialisten ausgeführt wird, aufzulösen. Die Erzählung von Martin spielt fast ausschließlich in der ideologischen Dimension, da er in einem Gespräch mit einem Nachbarn Attribuierungen in Bezug auf zurückliegende Ereignisse auf der Basis eines fest gefügten Weltbildes vornimmt. Erst mit dem ,frei beten“ kommt eine weitere Dimension ins Spiel. Zur religiösen Praxis äußert sich Martin jedoch nur sehr knapp, vermutlich, weil er dabei nicht aktiv beteiligt war.

Die vertiefende Interpretation belegt, dass die zentrale Spiritualitätskategorie der „Verbundenheit“ stark negativ geladen sein kann. Dabei kann die negative Verbundenheit im Sinne Buchers (2014) sowohl als horizontal (,,anderen schaden“, „Kälte- und Leerheitsgefühl“) als auch vertikal („Geister“, „schwarze Magie“, „ganz schlechte Energie“) klassifiziert werden. Dies spricht dafür, dass negative spirituelle Ladungen alle Bereiche des Spirituellen betreffen können. Daneben zeigt sich, dass verschiedene Dimensionen der Religiosität (Huber 2003) beim Umgang mit negativen spirituellen Phänomenen beteiligt sind. Auch dies spricht dafür, dass es sich dabei nicht um ein Randphänomen handelt.

\section{Diskussion}

Wie bereits festgehalten, können die vier Erzählungen sowohl horizontaler als auch vertikaler Verbundenheit im Spiritualitätsmodell von Bucher (2014) sowie unterschiedlichen Dimensionen der Religiosität nach Huber (2003) zugeordnet werden. Diese Variationsbreite spricht dafür, dass es sich nicht um singuläre und zufällige Phänomene handelt, sondern dass sie anthropologisch und soziologisch tiefer verankert sind. Dagegen kann eingewandt werden, dass derartige Erzählungen nur bei etwa $8 \%$ der Ausgangsstichprobe von 48 Fällen beobachtbar waren. Dem kann entgegengehalten werden, dass die Fälle nicht ausgesucht sind, sondern aus einer, nach theoretischen Kriterien gezogenen Zufallsstichprobe stammen, die auf einer repräsentativ angelegten Bevölkerungsumfrage basiert. Dies gibt den Fällen soziologisch ein größeres Gewicht. Dazu kommt, dass im Interviewleitfaden nicht nach negativen spirituellen Erfahrungen gefragt wurde. Vielmehr kann dem Interviewleitfaden im Erstinterview eher ein Priming in Bezug auf positive spirituelle Erfahrungen unterstellt werden, da vor der Frage zur Spiritualität der persönliche Umgang mit der Sinnfrage angesprochen wurde. Schließlich kann hervorgehoben werden, dass die vier Fälle aus einer Stichprobe von Säkularen stammen. Es kann vermutet werden, dass bei religiösen Auskunftspersonen, die mit einer spirituellen Welt vertrauter sind, auch mehr Erzählungen mit negativen spirituellen Erfahrungen vorkommen. Daher sollte die empirische Reichweite der vorliegenden Befunde nicht unterschätzt werden.

Zwei der vier Fälle (Antonio und Gabriel) lassen sich gut dem Formenkreis außergewöhnlicher Erfahrungen zuordnen (vgl. Bauer und Schetsche 2003; Belz 2009; Kohls 2004, 2016; Mayer und Kohls 2018; Schäfer 2012). In einer im Jahr 2000 durchgeführten, für Deutschland repräsentativen, Telefonbefragung $(n=1510)$ berichteten fast drei Viertel der Teilnehmenden mindestens ein außergewöhnliches 
Erlebnis, „das sich im weitesten Sinn dem Bereich übersinnlicher Erfahrungen zuordnen lässt" (Schmied-Knittel und Schetsche 2003, S. 33). Leider liegen keine Angaben vor, wie viele dieser Erlebnisse als bedrohlich oder in anderer Weise negativ interpretiert wurden. Wegen der Unerklärlichkeit, die mit derartigen Erlebnissen verbunden ist, kann jedoch vermutet werden, dass dies bei einer größeren Anzahl der Fall ist. Auch dies spricht dafür, dass die empirische Reichweite der vorliegenden Befunde nicht unterschätzt werden sollte.

Die berichteten Befunde legen nahe, negative spirituelle Erfahrungen systematisch und vertieft zu untersuchen. Dabei bietet es sich an, nicht zu schnell mit dem Begriff der Spiritualität zu operieren, da dieser im gesellschaftlichen Diskurs eher positiv besetzt ist und dies zu einem positiven Priming dieses Begriffs führt. Stattdessen sollten zunächst außergewöhnliche, paranormale und übersinnliche Erfahrungen angesprochen werden. Ein erster Schritt in diese Richtung könnte in einer Sekundäranalyse der 220 halbstrukturierten Interviews bestehen, die im Anschluss an die im vorangegangenen Absatz beschriebene, repräsentative Studie durchgeführt wurden. In diesem Material sollten auch negative Erlebnisse vorhanden sein. Ihre Auswertung könnte weitere Aufschlüsse über die innere Struktur und den Verbreitungsgrad negativer spiritueller Erfahrungen zur Verfügung stellen. Daneben wären Studien zu negativen spirituellen Erfahrungen in psychiatrischen Kontexten vielversprechend - vergleiche den Fall Martin. Dazu ist am Berner Institut für Empirische Religionsforschung bereits ein von Herbert Scheiblich geleitetes Projekt angelaufen.

Für derartige Studien ist ein Begriff der Spiritualität notwendig, der nicht auf positive Empfindungen und Effekte des spirituellen Erlebens und Verhaltens zentriert ist, sondern auch für die Möglichkeit negativer Empfindungen und Effekte offen ist. Dies kann unserer Ansicht nach erreicht werden, wenn die Definition von Spiritualität nicht die - durchaus deutlich vorhandenen - positiven psychohygienischen Korrelate von Spiritualität in den Vordergrund rückt, sondern stattdessen den Fokus auf die menschliche Fähigkeit der Selbsttranszendenz im Sinne des Begriffs der „exzentrischen Positionalität“ (Plessner 1928) und die Vergesellschaftung des Umgangs mit Transzendenzerfahrungen (Luckmann 1991) richtet. Spiritualität würde dann zum Begriff für Erfahrungen ,großer Transzendenzen“ im Sinne Luckmanns. Auf diese Weise bekäme auch die Debatte um die Verhältnisbestimmung von Spiritualität und Religion neue Impulse. Spiritualität könnte als eine, anthropologisch gesetzte und selbst noch nicht religiöse Quelle von Religion verstanden werden, die in der Interaktion und Verschmelzung mit religiösen Traditionen einen guten Teil des religiösen Erlebens immer wieder von Neuem fundiert. Spirituelle Erfahrung und religiöse Tradition würden dabei als zwei grundlegende und relativ unabhängige Quellen des Religiösen bestimmt. Darin hätten auch negative spirituelle Erfahrungen Platz und könnten mit entsprechenden religiösen Traditionen amalgamiert werden (vgl. den Fall Martin). Auf der anderen Seite ist es bei einer derartigen Verhältnisbestimmung immer noch möglich, dass Spirituelles sich nicht mit religiösen Traditionen amalgamiert und so außerhalb des Bereichs des Religiösen bleibt (vgl. die Fälle Antonio und Gabriel).

Funding Open access funding provided by University of Bern 
Open Access Dieser Artikel wird unter der Creative Commons Namensnennung 4.0 International Lizenz veröffentlicht, welche die Nutzung, Vervielfältigung, Bearbeitung, Verbreitung und Wiedergabe in jeglichem Medium und Format erlaubt, sofern Sie den/die ursprünglichen Autor(en) und die Quelle ordnungsgemäß nennen, einen Link zur Creative Commons Lizenz beifügen und angeben, ob Änderungen vorgenommen wurden.

Die in diesem Artikel enthaltenen Bilder und sonstiges Drittmaterial unterliegen ebenfalls der genannten Creative Commons Lizenz, sofern sich aus der Abbildungslegende nichts anderes ergibt. Sofern das betreffende Material nicht unter der genannten Creative Commons Lizenz steht und die betreffende Handlung nicht nach gesetzlichen Vorschriften erlaubt ist, ist für die oben aufgeführten Weiterverwendungen des Materials die Einwilligung des jeweiligen Rechteinhabers einzuholen.

Weitere Details zur Lizenz entnehmen Sie bitte der Lizenzinformation auf http://creativecommons.org/ licenses/by/4.0/deed.de.

\section{Literatur}

Altmeyer, S., C. Klein, B. Keller, C.F. Silver, R.W. Hood, und H. Streib. 2015. Subjective definitions of spirituality and religion. International Journal of Corpus Linguistics 20(4):526-552.

Bauer, E., und M. Schetsche. 2003. Alltägliche Wunder. Erfahrungen mit dem Übersinnlichen - wissenschaftliche Befunde. Würzburg: Ergon.

Belz, M. 2009. Außergewöhnliche Erfahrungen. Göttingen: Hogrefe.

Bucher, A.A. 2014. Psychologie der Spiritualität, 2. Aufl., Weinheim: Beltz.

Demmrich, S., und S. Huber. 2019. Multidimensionality of spirituality: a qualitative study among secular individuals. Religions 10(11):613. https://doi.org/10.3390/rel10110613.

Demmrich, S., und S. Huber. 2020. What do seculars understand as "spiritual"? A replication of Eisenmann et al.'s semantics of spirituality. Journal of Religion in Europe https://doi.org/10.1163/1874892913010008.

Eisenmann, C., C. Klein, A. Swhajor-Biesemann, U. Drexelius, B. Keller, und H. Streib. 2016. Dimensions of "spirituality": the semantics of subjective definitions. In Semantics and psychology of spirituality, Hrsg. H. Streib, R.W. Hood Jr., 125-151. Cham: Springer.

Emmons, R.A. 2000. Is spirituality an intelligence? Motivation, cognition, and the psychology of ultimate concern. The International Journal for the Psychology of Religion 10(1):3-26.

Huber, S. 2003. Zentralität und Inhalt. Ein neues multidimensionales Modell der Religiosität. Opladen: Leske + Budrich.

Koenig, H.G. 2008. Concerns about measuring "spirituality" in research. Journal of Nervous and Mental Disease 196:349-355.

Koenig, H.G. 2011. Spirituality and health research: Methods, measurement, statistics, and resources. West Conshohocken, PA: Templeton Foundation Press.

Kohls, N. 2004. Außergewöhnliche Erfahrungen - Blinder Fleck der Psychologie? Münster: LIT.

Kohls, N. 2016. Spiritualität und außergewöhnliche Erfahrungen im Kontext der akademischen Psychologie. In Spiritualität und spirituelle Krisen: Handbuch zu Theorie, Forschung und Praxis, Hrsg. L. Hofmann, P. Heise, 3-12. Stuttgart: Schattauer.

Luckmann, T. 1991. Die unsichtbare Religion. Berlin: Suhrkamp.

Mayer, C.-H., und N. Kohls. 2018. Zum Stand der Forschung zu ,außergewöhnlichen Erfahrungen“ und ihren psychologischen Auswirkungen. In Spiritualität in Psychiatrie \& Psychotherapie, Hrsg. G. Juckel, K. Hoffmann, und H. Walach, 237-250. Lengerich: Pabst.

Plessner, H. 1928. Die Stufen des Organischen und der Mensch. Einleitung in die philosophische Anthropologie. Berlin: de Gruyter.

Schäfer, C. 2012. Außergewöhnliche Erfahrungen - Konstruktion von Identität und Veränderung in autobiografischen Erzählungen. Münster: LIT.

Schmied-Knittel, I., und M. Schetsche. 2003. Psi-Report Deutschland. In Alltägliche Wunder. Erfahrungen mit dem Übersinnlichen - wissenschaftliche Befunde, Hrsg. E. Bauer, M. Schetsche, 13-38. Würzburg: Ergon.

Tong, Y., und F. Yang. 2018. Internal diversity among "spiritual but not religious" adolescents in the United States: a person-centered examination using latent class analysis. Review of Religious Research 60:435-453.

Zinnbauer, B.J., und K.I. Pargament. 2005. Religiousness and spirituality. In Handbook of the psychology of religion and spirituality, Hrsg. R.F. Paloutzian, C.L. Park, 21-42. New York: Guilford. 